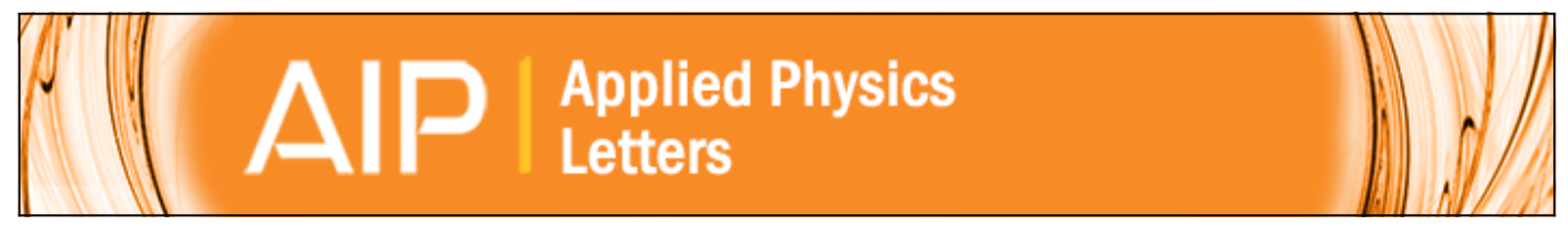

Magnetic field tuning of the effective $\mathbf{g}$ factor in a diluted magnetic semiconductor quantum dot

Kai Chang, J. B. Xia, and F. M. Peeters

Citation: Applied Physics Letters 82, 2661 (2003); doi: 10.1063/1.1568825

View online: http://dx.doi.org/10.1063/1.1568825

View Table of Contents: http://scitation.aip.org/content/aip/journal/apl/82/16?ver=pdfcov

Published by the AIP Publishing

Over 700 papers \&

presentations on

multiphysics simulation vew now

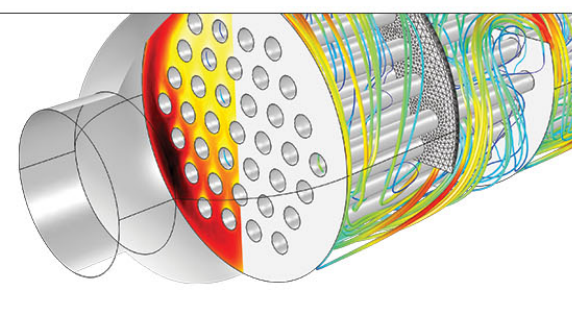




\title{
Magnetic field tuning of the effective $g$ factor in a diluted magnetic semiconductor quantum dot
}

\author{
Kai Chang ${ }^{\text {a) }}$ \\ NLSM, Institute of Semiconductor, Chinese Academy of Sciences, P. O. Box 912, Beijing 100083, China \\ and Department of Physics, University of Antwerp (UIA), B-2610 Antwerpen, Belgium \\ J. B. Xia \\ NLSM, Institute of Semiconductor, Chinese Academy of Sciences, P. O. Box 912, Beijing 100083, China \\ F. M. Peeters ${ }^{\text {b) }}$ \\ Department of Physics, University of Antwerp (UIA), B-2610 Antwerpen, Belgium
}

(Received 3 February 2003; accepted 24 February 2003)

\begin{abstract}
The spin interaction and the effective $g$ factor of a magnetic exciton (ME) are investigated theoretically in a diluted magnetic semiconductor (DMS) quantum dot (QD), including the Coulomb interaction and the $s p-d$ exchange interaction. At low magnetic field, the ME energy decreases rapidly with increasing magnetic field and saturates at high magnetic field for high $\mathrm{Mn}$ concentration. The ground state of the ME exhibits an interesting crossing behavior between $\sigma^{+}$-ME and $\sigma^{-}$-ME for low Mn concentration. The $g_{\text {ex }}$ factor of the ME in a DMS QD displays a monotonic decrease with increasing magnetic field and can be tuned to zero by an external magnetic field. (C) 2003 American Institute of Physics. [DOI: 10.1063/1.1568825]
\end{abstract}

The spin of carriers in semiconductor nanostructures has attracted recently considerable interest because of its importance for basic physics as well as for its potential application in spintronic devices. Recently, several quantum computational approaches ${ }^{1,2}$ based on semiconductor quantum dot systems were proposed due to the long spin-coherence time in semiconductors. ${ }^{3}$ Electron-hole entanglement involving two magnetoexciton states was recently identified experimentally in a single GaAs quantum dot (QD). ${ }^{4}$ The photon polarization and the spin of carriers in semiconductors are suitable candidates for quantum information storage and processing. The coherent transfer of quantum information among the different physical systems requires a quantum device, e.g. a photodetector, which can preserve the entanglement while the quantum information is transferred from the photon polarization to the exciton spin in the semiconductor. In order to maintain the entanglement, the quantum device should absorb equally into up $\left(\sigma^{+}\right)$and down $\left(\sigma^{-}\right)$exciton spin states, therefore the effective $g_{\text {ex }}$ factor of the exciton in the semiconductor has to be zero which should be realized by adjusting the parameters of the physical system.

The $g$ factor is important in understanding the spindependent optical and transport properties in semiconductor nanostructures. Since the Coulomb interaction, ${ }^{5}$ the quantum confinement effect, ${ }^{6}$ spin-orbit coupling, ${ }^{7}$ electron-hole exchange interaction, ${ }^{8}$ and hyperfine interaction ${ }^{9,10}$ can influence the spin splitting, therefore the $g$ factor of the electron, the hole and the exciton will provide us with insights in the spin relaxation and coherence in semiconductors. In a nonmagnetic semiconductor, the exchange interaction between the carriers and the nuclei of the host semiconductor material has been demonstrated experimentally although its strength

\footnotetext{
${ }^{a)}$ Electronic mail: kchang@red.semi.ac.cn

${ }^{b)}$ Electronic mail: peeters@uia.ua.ac.be
}

is rather weak $(\sim \mu \mathrm{eV}){ }^{9,10}$ In the diluted magnetic semiconductors (DMS) the $s p-d$ exchange interaction between the carriers and the magnetic impurities is strong which leads to a giant spin splitting, and spin-dependent transport, and optical properties. ${ }^{11-15}$ Very recently, a DMS QD was fabricated, using molecular beam epitaxy techniques, which was found to be more robust against thermal fluctuations. Photoluminescence experiments ${ }^{16-18}$ clearly demonstrated that the formation of a zero-dimensional magnetic exciton (ME) in the DMS QD leads to a suppression of the nonradiative recombination process.

In this work we investigate theoretically the $g_{\text {ex }}$ factor and the energy of the zero-dimensional ME in $\mathrm{Cd}_{1-x} \mathrm{Mn}_{x} \mathrm{Te} / \mathrm{Cd}_{1-y} \mathrm{Mg}_{y}$ Te DMS QDs, including the contribution of the Coulomb interaction and the $s p-d$ exchange interaction. We show how the effective $g_{\text {ex }}$ factor of the ME in the DMS QD can be engineered by using the fact that for low Mn concentration, the spin splitting caused by the $s p-d$ interaction is comparable to the intrinsic Zeeman splitting. For low Mn concentration the $g_{\text {ex }}$ factor of the ME decreases rapidly with increasing magnetic field at low magnetic fields and saturates at high magnetic field. The most interesting phenomenon is that the effective $g_{\text {ex }}$ factor of the ME can be tuned to zero by changing the magnetic field. This is extremely interesting for practical realization of, e.g., quantum information transfer from a photon system to excitons in a semiconductor system.

The effective $g_{\text {ex }}$ factor is determined by the spin splitting between the $\sigma^{+}$and $\sigma^{-}$polarized transition in DMS QDs:

$$
g_{\mathrm{ex}}=\Delta E_{\mathrm{ME}} / \mu_{B} B=g_{\mathrm{ex}}^{0}+g_{\mathrm{ex}}^{s p-d},
$$

where the Zeeman splitting $\Delta E_{\mathrm{ME}}=E_{\mathrm{ME}}\left(\sigma^{+}\right)-E_{\mathrm{ME}}\left(\sigma^{-}\right)$ $=\Delta E_{s p-d}^{ \pm}+\Delta E_{\text {Zeeman }}^{ \pm}$denotes the total spin splitting caused by the intrinsic Zeeman effect and the $s p-d$ exchange inter- 


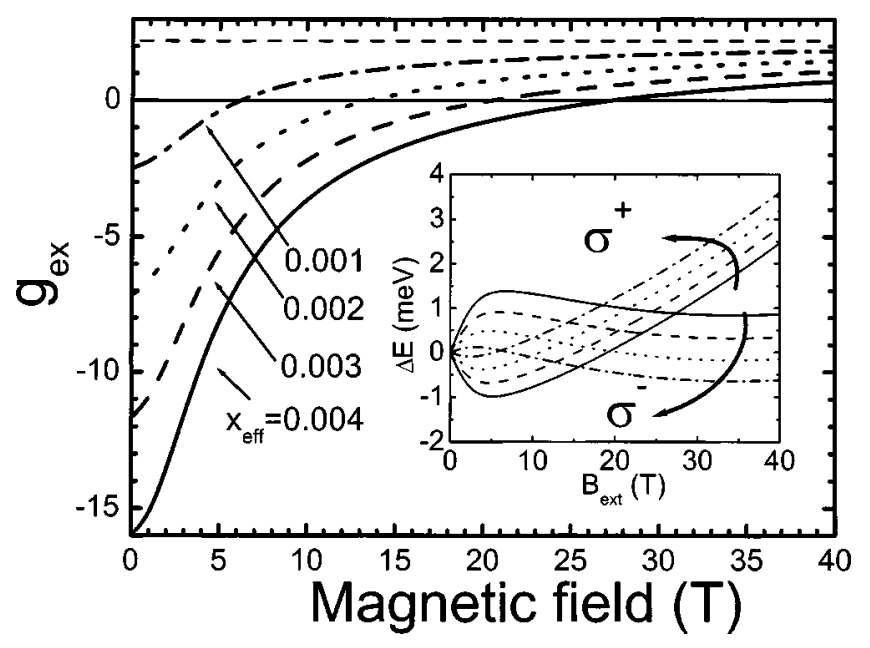

FIG. 1. The effective $g_{\text {ex }}$ factor and the ME energy (inset, the same line conventions are used as in the main figure) vs magnetic fields for low Mn concentrations. The quantum disk has $R=4 \mathrm{~nm}, h=1 \mathrm{~nm}$, and the results are shown for $T=2 \mathrm{~K}$. The magnetic field where $g_{\mathrm{ex}}=0$ defines the critical magnetic field $B_{c}$.

action for spin-up or spin-down $\mathrm{ME}$, where $E_{\mathrm{ME}}\left(\sigma^{ \pm}\right)$is the energy of the spin-up or spin-down ME, i.e., two possible transitions with different circular polarization (see lower inset of Fig. 1). $g_{\mathrm{ex}}^{0}$ is the intrinsic $g$ factor, and $g_{\mathrm{ex}}^{s p-d}$ the $g$ factor induced by the $s p-d$ exchange interaction. The energy of the zero-dimensional ME in a DMS disk-like quantum dot embedded in a nonmagnetic semiconductor material can be obtained from the Schrödinger equation $H_{\mathrm{ME}} \Psi_{\mathrm{ME}}\left(\mathbf{r}_{e}, \mathbf{r}_{h}\right)=E_{\mathrm{ME}} \Psi_{\mathrm{ME}}\left(\mathbf{r}_{e}, \mathbf{r}_{h}\right)$. Here, the stationary ME Hamiltonian $H_{\mathrm{ME}}^{\sigma \pm}$ for the $\sigma^{ \pm}$ME in the DMS QD is

$$
\begin{aligned}
H_{\mathrm{ME}}^{ \pm}= & \frac{1}{2 m_{e}^{*}}\left[\mathbf{p}_{e}+e \mathbf{A}\left(\mathbf{r}_{e}\right)\right]^{2}+V_{e}\left(\rho_{e}, z_{e}\right)+g_{e}^{*} \mu_{B} B \sigma_{z} \\
& +\frac{1}{2 m_{h}^{*}}\left[\mathbf{p}_{h}-e \mathbf{A}\left(\mathbf{r}_{h}\right)\right]^{2}+V_{h}\left(\rho_{h}, z_{h}\right)+g_{h}^{*} \mu_{B} B j_{z} \\
& +V_{\text {exch }}-\frac{e^{2}}{\epsilon|\mathbf{r}|},
\end{aligned}
$$

where $\mathbf{r}=\mathbf{r}_{e}-\mathbf{r}_{h}=(\rho, z)$ denotes the electron-hole relative coordinates, $m_{e}\left(m_{h}\right)$ is the effective mass of electron (hole), $\epsilon$ is the dielectric constant and $\sigma_{z}= \pm 1 / 2\left(j_{z}= \pm 1 / 2, \pm 3 / 2\right)$ is the electron (hole) spin. The band mixing is neglected due to the strong confinement along the growth direction. $V_{e}\left(V_{h}\right)$ is the confining potential of electron (hole) in the QD, i.e., $V_{e(h)}\left[z_{e(h)}\right]=0$ inside the QD and $V_{e(h)}\left[z_{e(h)}\right]=V_{e(h)}$ otherwise. $g_{e}^{*}\left(g_{h}^{*}\right)$ is the effective Lande $g$ factor of electron (hole). The $s p-d$ exchange interaction term $V_{\text {exch }}$ between the carriers and the magnetic ion $\mathrm{Mn}^{2+}$ is treated in a meanfield approximation ${ }^{11}$

$$
V_{\text {exch }}=J_{s-d}\left\langle S_{z}\right\rangle \sigma_{z}+J_{p-d}\left\langle S_{z}\right\rangle j_{z}
$$

where $J_{s-d}=-N_{0} \alpha x_{\mathrm{eff}}, \quad J_{p-d}=-N_{0} \beta x_{\mathrm{eff}} / 3, \quad$ and $\left\langle S_{z}\right\rangle$ $=S_{0} B_{J}\left[S g_{\mathrm{Mn}} \mu_{B} B / k_{B}\left(T+T_{0}\right)\right], S=5 / 2$ corresponds to the spins of the localized $3 d^{5}$ electrons of the $\mathrm{Mn}^{2+}$ ions. $B_{J}(x)$ is the Brillouin function, $N_{0}$ is the number of cations per unit volume, the reduced effective concentration of $\mathrm{Mn}$ is given by the phenomenological parameter $x_{\text {eff }}$, and $T_{0}$ accounts for the reduced single-ion contribution due to the antiferromag- netic Mn-Mn coupling, $k_{B}$ is the Boltzmann constant, $\mu_{B}$ is the Bohr magneton, $g_{\mathrm{Mn}}=2$ is the $g$ factor of the $\mathrm{Mn}^{2+}$ ion. The total magnetic field is

$$
B=B_{\mathrm{ME}} \pm B_{\mathrm{ext}},
$$

where $B_{\text {ext }}$ is the external magnetic field, and the carrierinduced exchange field ${ }^{16}$ inside $\mathrm{ME}$ is proportional to the squared wave function of the carriers, i.e., $B_{\mathrm{ME}}$ $\approx\left(1 / 3 \mu_{B} g_{\mathrm{Mn}}\right) \beta j_{z}\left|\phi_{h}(r)\right|^{2}$. The ME wave function with total angular momentum $L$ is constructed as a linear combination of the single particle eigenstates

$\Psi_{\mathrm{ex}}^{L}\left(\mathbf{r}_{e}, \mathbf{r}_{h}\right)=\sum_{n, k, l_{1}, l_{2}, l_{1}+l_{2}=L} a_{n k}^{l} \phi_{n, l_{1}}^{e}\left(\rho_{e}, z_{e}\right) \phi_{k, l_{2}}^{h}\left(\rho_{h}, z_{h}\right)$

The single-particle eigenstates of the electron $\left(\phi_{n, l_{1}}^{e}\left(\rho_{e}, z_{e}\right)\right]$ and the hole $\left[\phi_{k, l_{2}}^{h}\left(\rho_{h}, z_{h}\right)\right]$ are obtained by solving the stationary Schrödinger equation using the finite difference method. ${ }^{19}$ More detailed information about the nonuniform space grid can be found in Ref. 19. In our calculation, the QD is modeled as a disk (see the left upper inset in Fig. 1) and we take $n=10$ and $k=10$ [see Eq. (5)] which leads to an accuracy for the ground state energy better than $1 \%$. The transition energy of $\mathrm{ME}$ for both $\sigma^{+}$and $\sigma^{-}$excitation is $E=E_{g}(T)+E_{\mathrm{ME}}^{ \pm}$, where $E_{g}(T)=E_{g}(0)-a T^{2} /(b+T)$ is the semiconductor band gap which depends on the temperature, the parameters $a=0.346$ and $b=15.059$ are obtained by fitting the band gap $E_{g}(T)$ at low temperature, and $d E_{g}(T) / d T$ is obtained from these parameters at $T=77 \mathrm{~K}$, which agrees well with the previous experimental results (see Ref. 20), $\left.d E_{g}(T) / d T \approx-3 \times 10^{-4} \mathrm{eV} / \mathrm{K}\right) . E_{\mathrm{ME}}^{+}\left(E_{\mathrm{ME}}^{-}\right)$is the $\mathrm{ME}$ energy for the $\sigma^{+}\left(\sigma^{-}\right)$transition, respectively. The parameters used in our calculations are $m_{e}^{*}=0.096 m_{0}, m_{h}=0.6 m_{0}, m_{0}$ is the free electron mass. $x_{\text {eff }}=0.045, g_{\mathrm{Mn}}=2, N_{0} \alpha=0.22 \mathrm{eV}$, $N_{0} \beta=-0.88 \quad \mathrm{eV}, \quad S_{0}=1.32, \quad T_{0}=3.1 \quad \mathrm{~K}^{16}{ }^{16} g_{e}^{*}=-1.47$, $g_{h}^{*}=-0.24,{ }^{21} \quad g_{\mathrm{ex}}^{0}=\left(g_{e}^{*}+3 g_{h}^{*}\right) \approx 2.2, \quad \epsilon=10.6, \quad E_{g}=1.586$ $+1.51 x(\mathrm{eV})$ for $\mathrm{Cd}_{1-x} \mathrm{Mn}_{x} \mathrm{Te}$, and $E_{g}=1.586+1.705 y(\mathrm{eV})$ for $\mathrm{Cd}_{1-y} \mathrm{Mg}_{y} \mathrm{Te}^{22}$

Figure 1 depicts the variation of the $g_{\mathrm{ex}}$ factor and the energy (the inset of Fig. 2) of the ME with magnetic field for low Mn concentrations. The $g_{\text {ex }}$ factor shows a monotonic increase with increasing magnetic field for different $\mathrm{Mn}$ concentrations. It is important to notice that the $g_{\text {ex }}$ factor can be tuned to zero by changing the magnetic field, i.e., the energies of $\sigma^{ \pm}$ME become degenerate at these magnetic fields $B_{c}$. This behavior can be understood from the inset which shows the ME energy as a function of magnetic field. From the inset, one finds that the variation of the ME energy versus magnetic field is different from that in DMS QD with high Mn concentration (see Ref. 16). For the DMS QD with low Mn concentration, the spin splitting $\Delta E_{s p-d}$ induced by the $s p-d$ interaction is comparable to the intrinsic Zeeman splitting $\Delta E_{\text {Zeeman }}$. Due to the competition between these interactions, the ground state of the ME energy changes from $\sigma^{+}$ ME to $\sigma^{-}$ME, i.e., the polarization of the emission light changes from $\sigma^{+}$circular polarization to $\sigma^{-}$circular polarization with increasing magnetic field. This interesting crossing behavior is only possible when the sign of the spin splitting $\Delta E_{s p-d}$ is opposite to the intrinsic Zeeman splitting $\Delta E_{\text {Zeeman. }}$. The significant variation of the $g_{\text {ex }}$ factor with 


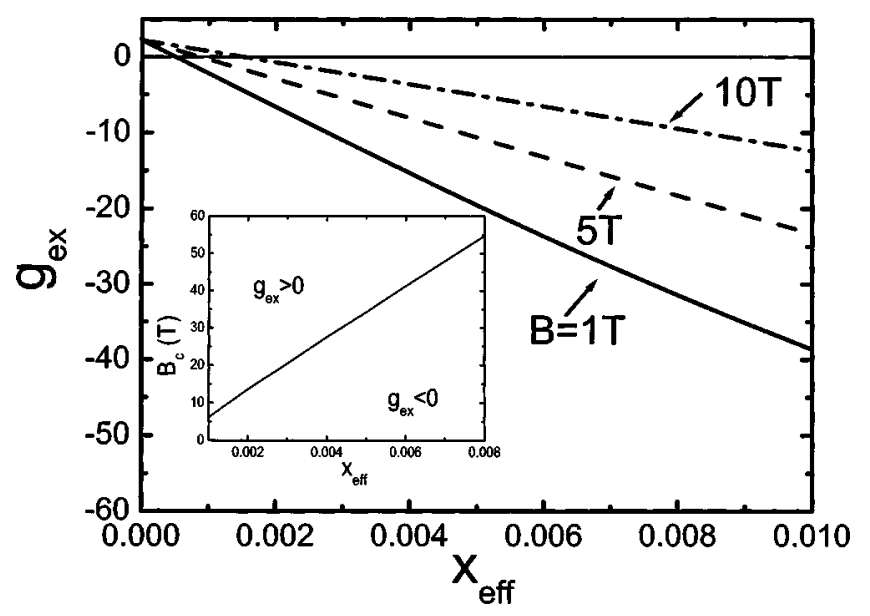

FIG. 2. The effective $g_{\text {ex }}$ factor and the critical magnetic field $B_{c}$ (inset) vs the effective Mn concentration for a quantum disk with $R=4 \mathrm{~nm}, h=1 \mathrm{~nm}$ at a temperature $T=2 \mathrm{~K}$.

magnetic field provides us with a freedom to tailor the $\mathrm{ME}$ transition energies, and its polarization, in semiconductors.

In Fig. 2 we plot the $g_{\text {ex }}$ factor as a function of the effective Mn concentration $x_{\text {eff }}$ in a DMS QD for different magnetic fields. Two characteristics can be found in this figure: (i) the $g_{\mathrm{ex}}$ factor of $\mathrm{ME}$ is initially equal to $g_{\mathrm{ex}}^{0}=2.2$, i.e., the intrinsic $g_{\text {ex }}$ factor, decreases almost linearly with increasing Mn concentration, and (ii) decreases with increasing magnetic field. The first characteristic arises from the fact that the spin splitting induced by the $s p-d$ exchange interaction increases linearly with increasing effective Mn concentration [see Eq. (3)]. The second characteristic can be understood from the dependence of the ME energy on the magnetic field (see Fig. 1). Because of the competition between the intrinsic Zeeman effect and the $s p-d$ exchange interaction a crossing occurs between the $\sigma^{+} \mathrm{ME}$ and the $\sigma^{-}$ $\mathrm{ME}$, i.e., the spin splitting of the $\sigma^{ \pm} \mathrm{ME}$ decreases with increasing magnetic field. The inset shows the critical magnetic field $B_{c}$ versus the effective Mn concentration $x_{\text {eff }}$. The line indicates the zero $g_{\text {ex }}$ factor at critical magnetic fields, below the line $g_{\text {ex }}<0$ and above the line $g_{\text {ex }}>0$.

Recent experiments have shown that a magnetic field parallel to the hole quantization axis (Faraday geometry) suppresses strongly the magnetic fluctuations in DMS QD. ${ }^{23}$ Therefore, the mean-field approximation adopted in our calculation $^{11}$ in which the fluctuation effect of the magnetic ions is neglected, is expected to be a good first-order approximation. The effect of fluctuation will be enhanced for low Mn concentration and higher temperature, which well lead to a higher critical magnetic field $B_{c}$. Nevertheless the underlying physics picture of the magnetic field tuning of $g_{\mathrm{ex}}$ will stay valid.

In summary, we investigated the spin interaction and the effective $g_{\text {ex }}$ factor of a ME in a DMS QD for different magnetic fields and Mn concentrations. The effective $g_{\text {ex }}$ factor of the ME in a DMS QD can be tuned to zero by a magnetic field for low Mn concentration. The variation of $g_{\text {ex }}$ and the $\sigma^{ \pm}$ME energy with magnetic field are quite different for different Mn concentrations. The exchange interaction in the DMS QD provides us with a freedom to tailor the spin interaction and the effective $g_{\text {ex }}$ factor of the semiconductor quantum dot by varying the external magnetic field and the Mn concentration.

This work was partly supported by the NSF of China, the special fund for Major State Basic Research Project No. G001CB3095 of China, Nano Science Foundation from CAS, the Flemish Science Foundation (FWO-Vl), and the Bilateral Cooperation program between Flanders and China.

${ }^{1}$ B. E. Kane, Nature (London) 393, 133 (1998).

${ }^{2}$ D. Loss and D. P. DiVincenzo, Phys. Rev. A 57, 120 (1998).

${ }^{3}$ J. M. Kikkawa, I. P. Smorchkova, N. Samarth, and D. D. Awshalom, Science 277, 1284 (1997); J. A. Gupta, D. D. Awschalom, X. Peng, and A. P. Alivisatos, Phys. Rev. B 59, R10421 (1999).

${ }^{4}$ G. Chen, N. H. Bonadeo, D. G. Steel, D. Gammon, D. S. Katzer, D. Park, and L. J. Sham, Science 289, 1906 (2000).

${ }^{5}$ M. Oestreich and W. W. Rühle, Phys. Rev. Lett. 74, 2315 (1995).

${ }^{6}$ M. Bayer, V. B. Timofeev, T. Gutbrod, A. Forchel, R. Steffen, and J. Oshinowo, Phys. Rev. B 52, R11623 (1995); W. Heller and U. Bockelmann, ibid. 55, 4871 (1997); A. A. Kiselev, E. L. Ivchenko, and U. Rössler, ibid. 58, 16353 (1998).

${ }^{7}$ M. Willatzen, M. Cardona, and N. E. Christensen, Phys. Rev. B 51, 17992 (1995).

${ }^{8}$ D. Gammon, E. S. Snow, B. V. Shanabrook, D. S. Katzer, and D. Park, Phys. Rev. Lett. 76, 3005 (1996); Science 277, 85 (1997); U. Woggon, F. Gindele, W. Langbein, and M. Hetterich, Phys. Status Solidi A 164, 505 (1997).

${ }^{9}$ D. Gammon, Al. L. Efros, T. A. Kennedy, M. Rosen, D. S. Katzer, D. Park, S. W. Brown, V. L. Korenev, and I. A. Merkulov, Phys. Rev. Lett. 86, 5176 (2001)

${ }^{10}$ J. H. Smet, R. A. Deutschmann, F. Ertl, W. Wegschelder, G. Abstreiter, and K. von Klitzing, Nature (London) 415, 281 (2002).

${ }^{11}$ J. K. Furdyna, J. Appl. Phys. 64, R29 (1988), and references therein.

${ }^{12}$ D. D. Awschalom, J. Warnock, and S. von Molnar, Phys. Rev. Lett. 58, 812 (1987).

${ }^{13}$ H. Krenn, K. Kaltenegger, T. Dietl, J. Spalek, and G. Bauer, Phys. Rev. B 39, 10918 (1989).

${ }^{14}$ V. V. Rossin, F. Henneberger, and J. Puls, Phys. Rev. B 53, 16444 (1996).

${ }^{15}$ P. A. Wolff, in Semiconductors and Semimetals, Diluted Magnetic Semiconductors, edited by J. K. Furdyna and J. Kossut (Academic, New York, 1988), Vol. 25, p. 413.

${ }^{16}$ A. A. Maksimov, G. Bacher, A. MacDonald, V. D. Kulakovskii, A. Forchel, C. R. Becker, G. Landwehr, and L. Molenkamp, Phys. Rev. B 62, R7767 (2000).

${ }^{17}$ S. H. Xin, P. D. Wang, A. Yin, C. Kim, M. Dobrowolska, J. L. Merz, and J. K. Furdyna, Appl. Phys. Lett. 69, 2884 (1996).

${ }^{18}$ G. Bacher, H. Schomig, M. K. Welsch, S. Zaitsev, V. D. Kulakovskii, A. Forchel, S. Lee, M. Dobrowolska, J. K. Furdyna, B. Konig, and W. Ossau, Appl. Phys. Lett. 79, 524 (2001).

${ }^{19}$ F. M. Peeters and V. A. Schweigert, Phys. Rev. B 53, 1468 (1996).

${ }^{20}$ Physics of II-VI and I-VII CoMEounds, Semimagnetic Semiconductors, Landolt-Börnstein, New Series, Group III Vol. 17b, edited by O. Madelung, M. Schulz, and H. Weiss (Springer, Berlin, 1982).

${ }^{21}$ S. Takeyama, G. Karczewski, T. Wojtowicz, J. Kossut, H. Kunimatsu, K. Uchida, and N. Miura, Phys. Rev. B 59, 7327 (1999).

${ }^{22}$ J. A. Gai, R. R. Galazka, and Nawrocki, Solid State Commun. 25, 193 (1978); Superlattices Microstruct. 16, 5 (1994).

${ }^{23}$ G. Bacher, A. A. Maksimov, H. Schromig, V. D. Kulakovskii, M. K. Welsch, A. Forchel, P. S. Dorozhkin, A. V. Chernenko, S. Lee, M. Dobrowolska, and J. K. Furdyna, Phys. Rev. Lett. 89, 127201 (2002). 